\title{
GENETICS OF NON SYNDROMIC HEARING LOSS IN THE REPUBLIC OF MACEDONIA
}

\author{
Sukarova Stefanovska E ${ }^{1}$, Cakar $\mathrm{M}^{2}$, Filipce I², Plaseska Karanfilska D ${ }^{1}$
}

\begin{abstract}
*Corresponding Author: Emilija Sukarova Stefanovska, Ph.D., Research Centre for Genetic Engineering and Biotechnology "Georgi D. Efremov", Macedonian Academy of Sciences and Arts, Av. Krste Misirkov 2, POB 428, 1000 Skopje, Republic of Macedonia; Tel.: +389(0)2-3235-410; Fax: +389-(0)2-3115-434; E-mail: emilija@manu.edu.mk
\end{abstract}

\begin{abstract}
Hearing impairment is the most common sensory deficit in humans affecting 1 in 1000 newborns. When present in an infant, deafness may have dramatic effects on language acquisition, seriously compromising the quality of their life. Deafness is influenced by both genetic and environmental factors, with inherited causes as the most prominent etiological factor in deafness in developed countries. The genetic basis of hearing loss is complex with numerous loci and genes underlying hereditary sensoryneural non syndromic hearing loss (NSHL) in humans. Despite the wide functional heterogeneity of the genes, mutations in the GJB2 gene are found to be the most common cause of sporadic and recessive NSHL in many populations worldwide. Molecular characterization of deafness in the Republic of Macedonia was performed in 130 NSHL profoundly deaf children from different ethnic origins. Molecular studies included direct sequencing of the $G J B 2$ gene and specific polymerase chain reaction (PCR) analyses for the del(GJB6D13S1830) mutation. Five common mitochondrial DNA (mtDNA) mutations [A1555G, 961 delT+ C(n),

1 Research Centre for Genetic Engineering and Biotechnology "Georgi D. Efremov," Macedonian Academy of Sciences and Arts, Skopje, Republic of Macedonia

2 Othorinolaryngology Clininc, Clinical Center University Hospital, Skopje, Republic of Macedonia
\end{abstract}

T1095C, C1494T and A827G] were also analyzed using the SNaPShot method. In preliminary studies, GJB2 gene mutations were found in $36.4 \%$ of analyzed patients, with predominance of $35 \mathrm{delG}$ in Macedonian and Albanian patients and W24X in Gypsy patients, respectively. No $\operatorname{del}(G J B 6-$ D13S1830) mutation was found. None of the analyzed deafness-associated mutations in mtDNA were identified in the studied patients.

Keywords: DFNB1 locus, GJB2 gene mutations, Non syndromic hearing loss (NSHL).

\section{INTRODUCTION}

Hearing impairment is the most common sensory deficit in humans. Approximately 1 in 1000 newborns is affected by severe to profound deafness at birth, and additionally 1 in 1000 children progressively develops hearing loss during childhood. With the age, hearing loss progressively increases, so that over $50.0 \%$ of individuals above 80 years of age are affected [1]. The World Health Organization (WHO) has estimated that in 2005, 278 million people worldwide have moderate to profound hearing loss (WHO; http://www.who. $\mathrm{int} /$ mediacentre/factsheets/fs300/ en/index.html ). In the Republic of Macedonia, there are 9000 deaf individuals. When present in an infant, deafness may have dramatic effect on language acquisition, seriously compromising the quality of their life. 
Deafness is influenced by both genetic and environmental factors, with inherited causes defined as the most prominent etiological factor in deafness in developed countries. When associated with other symptoms it is referred to as syndromyc hearing loss (SHL), while when it occurs as an isolated symptom, it is referred to as non syndromic hearing loss (NSHL). Non syndromic hearing loss accounts for about $70.0 \%$ of genetic deafness and is almost exclusively monogenic and highly heterogeneous $[2,3]$. Non syndromic hearing loss can be transmitted as autosomal recessive $(80.0 \%)$, autosomal dominant $(20.0 \%)$, X-linked deafness and in mitochondrial mode $(2.0 \%)$. The autosomal recessive forms of deafness are generally the most severe and are almost exclusively caused by cochlear defects of hair cells (sensoryneural deafness). Autosomal dominant forms of deafness are usually post-lingual and progressive.

The genetic basis of hearing loss is complex. At least $1.0 \%$ of human protein-coding genes are involved in sound perception. There are approximately 113 mapped loci and 51 different genes that have been shown to underlie hereditary sensoryneural NSHL in humans (http:webhost.ua.ac. be/hhh/). They encode a wide variety of protein classes such as transcription factors, cytoskeletal and extracellular matrix components, and ion channels. Mutations in different genes can lead to the same clinical manifestation of deafness. Alternatively, different mutations in the same gene may result in syndromic, non syndromic, recessive or dominant deafness; finally, the same mutation can be associated with quite variable phenotypes [4].

Despite the wide functional heterogeneity of the genes involved in the perception of sound, mutations in the DFNB1 locus on chromosome 13 (13q12) are responsible for more than half of all cases of NSHL. The locus contains three genes, GJB2, GJB6 and GJA1, encoding for the transmembrane gap junction proteins connexin 26 , connexin 30 and connexin 31 , respectively, involved in ion transfer and homeostasis in the inner ear $[5,6]$.

Mutations in the GJB2 gene are the most common cause of sporadic and recessive NSHL, in many populations worldwide [5]. More than 100 different mutations in this gene have been described with specific prevalence in different ethnic groups and geographic regions. In Caucasians, the most com- mon mutation is 35delG [7], 167delT in Ashkenazi Jews [8], 235delC in Japanese [9], while W24X is frequent in India and in Gypsies. Due to the high incidence of $G J B 2$ gene mutations, molecular testing for $G J B 2$ gene mutations has rapidly become the standard of care for the diagnosis and counseling of patients with non syndromic hearing impairment of unknown cause.

Molecular Characterization of Deafness in the Republic of Macedonia. Molecular characterization of deafness in the Republic of Macedonia started in 2006, as a project of the Macedonian Academy of Sciences and Arts (MASA) in collaboration with the Audiology Centre at the Clinic of Othorinolaryngology, Medical Faculty, Skopje. Until now, 130 unrelated cases of different ethnic origins [Macedonians (78), Albanians (19), Gypsies (30) and Turks (3)], all with profound NSHL, were ascertained through the Audiology Centre, where audiological examinations and detailed family history analyses were performed.

GJB2 Gene Mutation Analysis. All patients and members of their families were screened for the presence of the GJB2 gene mutations using single strand conformation polymorphism analysis, restriction enzyme digestion or direct sequencing of the coding exon 2. Multiplex ligation-dependent probe amplification (MLPA) analysis using SALSA MLPA kit P163-C1 Hearing loss probemix (MRC Holland, Amsterdam, The Netherlands) was performed in order to determine the presence of deletions/duplications in the $13 \mathrm{q}$ region, as well as the specific point mutations in the GJB2 gene (splice site mutation IVS1+1G $>$ A, 35delG, 101T $>C$, 167delT, 235delC and 313del14).

Preliminary studies on mutations in the GJB2 gene in 33 persons with pre lingual non syndromic deafness in the Republic of Macedonia determined a prevalence of $36.4 \%$, with $35 \mathrm{delG}$ as the most frequent variation found in $68.2 \%$ of mutated chromosomes, followed by W24X (18.2\%), predominantly in Gypsy families, V37I (9.1\%) and R127H (4.5\%) [10]. Additionally two other mutations were found in the analyzed patients, Cd120delGAG and T175I, each with a frequency of $0.8 \%$. None of the analyzed patients carry the IVS-I-1 (G>A) mutation. These findings indicate that as in other Caucasian populations, the $35 \mathrm{delG}$ mutation is the most frequent cause of deafness in the Republic of Macedonia and 
should be tested in each routine diagnostic approach in the Macedonian population, while the W24X mutation is the first mutation that should be tested in the deaf Macedonian Gypsy population.

Another member of the connexin family, connexin 30 , is encoded by the GJB6 gene located 350 $\mathrm{kb}$ upstream of $G J B 2$ within the same DFNB1 locus. One large deletion, del(GJB6-D13S1830), has been reported as a common cause of non syndromic deafness, truncating the GJB6 gene and also abolishing $G J B 2$ expression, possibly by deleting a currently unidentified GJB2 regulatory element [11]. This deletion, either in the homozygous state or in combination with a GJB2 gene mutation, has been described as the cause of non syndromic deafness at $5.0-9.0 \%$ of all DFNB1 alleles in Europe [12]. Screening for the presence of del(GJB6-D13S1830) in the GJB6 gene revealed no mutation in our group of analyzed patients.

Mitochondrial DNA Mutations Analysis. Mutations in mitochondrial DNA (mtDNA) are found to contribute to sensoryneural deafness, including both syndromic and non syndromic forms, with a variable frequency in different populations of deaf persons [13]. Hot-spot regions for deafness mutations are the MT-RNR1 gene, encoding the $12 \mathrm{~S}$ ribosomal RNA (rRNA) and the MT-TS1 gene, encoding the mitochondrial transfer RNA (tRNA) serine 1 . One hundred and thirty Macedonian patients with NSHL were screened for the five mitochondrial mutations associated with deafness $(\mathrm{A} 827 \mathrm{G}, 961 \mathrm{delT}+\mathrm{Cn}, \mathrm{T} 1095 \mathrm{C}, \mathrm{C} 1494 \mathrm{~T}$ and A1555G) by a single nucleotide primer extension assay utilizing the ABI PRISM ${ }^{\mathrm{TM}} \mathrm{SNaPshot}$ Multiplex Kit (Life Technologies, Carlsbad, CA, USA) following the manufacturer's instructions [14]. None of the analyzed deafness-associated mutations were identified in the studied patients.

These findings suggest that mtDNA mutations do not represent risk factor for sensoryneural deafness in the Macedonian population.

\section{REFERENCES}

1. Cohen MM, Gorlin RJ. Epidemiology, etiology and genetic patterns. In: Gorlin RJ, Toriello HV, Cohen MM, Eds. Hereditary Hearing Loss and Its Syndromes. Oxford: Academic Press. 1995: 9-21.
2. Schrijver I. Hereditary non-syndromic sensorineural hearing loss. Transforming silence to sound. J Molec Diagn. 2004; 6(4): 275-284.

3. Petit C. From deafness genes to hearing mechanisms: harmony and counterpoint. Trends Mol Med. 2006; 12(2): 57-64.

4. Mese G, Londin E, Mui R, Brink PR, White TW. Altered gating properties of functional Cx26 mutants associated with recessive non-syndromic hearing loss. Hum Genet. 2004; 115(3): 191-199.

5. Rabionet $\mathrm{R}$, Zelante $\mathrm{L}$, Lopez-Bigas $\mathrm{N}$, et al. Molecular basis of childhood deafness resulting from mutations in the GJB2 (Connexin 26) gene. Hum Genet. 2000; 106(1): 40-44.

6. Kennenson A, Van Naarden Brown K, Boyle C. GJB2 (Connexin 26) variants and nonsyndromic sensorineural hearing loss: A HuGE review. Genet Med. 2002; 4(4): 258-274.

7. Zelante I, Gasparini P, Estivill X, et al. Connexin 26 mutations associated with the most common form of non-syndromic neurosensory autosomal recessive deafness (DFNB1) in Mediterraneans. Hum Mol Genet. 1997; 6(9): 1605-1609.

8. Morell RJ, Kim HJ, Hood LJ, et al. Mutations in the connexin 26 gene (GJB2) among Ashkenazi Jews with nonsyndromic recessive deafness. N Engl J Med. 1998; 339(21): 1500-1505.

9. Abe S, Kelly PM, Kimberling WJ, Usami SI. Connexin 26 gene (GJB2) mutation modulates the severity of hearing loss associated with the $1555 \mathrm{~A}>\mathrm{G}$ mitochondrial mutation. Am J Med Genet. 2001; 103(4): 334-338.

10. Sukarova Stefanovska E, Momirovska A, Cakar M, Efremov GD. GJB2 mutations in non syndromic hearing loss in the Republic of Macedonia. Balkan J Med Genet. 2009; 12(2): 11-17.

11. Del Castillo I, Villamar M, Moreno-Pelayo MA, et al. A deletion involving the connexion 30 gene in nonsyndromic hearing impairment. N Engl J Med. 2002; 346(4): 243-249.

12. Del Castillo I, Moreno-Pelayo MA, Del Castillo FJ, et al. Prevalence and evolutionary origins of the del(GJB6-D13S1830) mutation in the DFNB1 locus in hearing-impaired subjects: a multi-center study. Am J Hum Genet. 2003; 73(6): 1452-1458.

13. Maniglia LP, Bruna Moreira CL, Menezes da Silva MA, Piatto VB, Maniglia JV. Screening of the mitochondrial A1555G mutation in patients with sensorineural hearing loss. Rev Bras Otorinolaryngol. 2008; 74(5): 731-736.

14. Bardien S, Human H, Harris T, et al. A rapid method for detection of five known mutations associated with aminoglycoside-induced deafness. BMC Med Genet. 2009; 10: 2. 